\title{
Female Sexual Dysfunction in Patients with Chronic Low Back Pain I.Y.Abdallah ${ }^{1}$, E.M.Youssef ${ }^{2}$ and Sahar M.Tantawy ${ }^{1}$ \\ ${ }^{1}$ Dermatology and Andrology, Dept., Faculty of Medicine, Benha Univ., Benha, Egypt \\ ${ }^{2}$ Neurosurgery, Dept., Faculty of Medicine, Zagazig Univ., Zagazig, Egypt
}

E-Mail:

\begin{abstract}
Facts: Female sexual dysfunction (FSD) is a common yet misdiagnosed condition. The purpose of this study was to examine this problem in Egypt and explain the effects of CLBP on the sexual lives of women. Methods: 200 married women with persistent low back pain participated in this cross-sectional research. All patients had a thorough history and clinical examination, as well as a 25-question self-report questionnaire with additional questions tailored to the study's objectives. English and Arabic are used to write this piece. To guarantee the privacy and security of all collected data. Results: $35 \%$ of the 30-39-year-old individuals had a positive history of FGC, and 54.8 percent had no history of FGC. While 33.7\% of individuals who had never had FGC and no one with FGC had frequency from 3 to 4 times, there was a statistically significant relationship between the patients' age, marital age and frequency of sex. FGC exposure reduced frequency in $18.5 \%$ of participants, who reported satisfaction with the frequency of intercourse and frequency of wetting in the bed during intercourse ( 88 percent and 63 percent of participants unexposed and exposed respectively get wetduring intercourse in more than 50 percent of sexual encounters). Conclusion: 54.8 percent of female chronic low back pain patients had female sexual dysfunction. Chronic low back pain was linked to decreased female erections.
\end{abstract}

Keywords: Sexual, Dysfunction, Chronic, Back, Pain.

\section{Introduction}

One of the most debilitating conditions in the world is low back pain. To a large extent, the severity of pain and impairment is determined by the extent to which degenerative changes have occurred in one of the spine's many components, such as the back muscles. When the intervertebral disc, vertebral endplates, spinal bodies and osseous elements, neural canal, facet joints and ligamentum flavum are altered, symptoms of low back pain can occur. Furthermore, changes in one of these elements can lead to subsequent degenerative changes in the other elements that make up the structural bony, ligamentous and muscular components of the spine [1].

Another important aspect of human existence is sexuality, which is seen as integral to overall well-being and the durability of close personal relationships. The sexual act is reliant on the interplay of many bodily systems, particularly the lumbar and pelvic regions' integrity and function [2].

Age, health, and personal experience all play a role in sexuality, which is a multifaceted process that includes societal and religious views as well. Sexual dysfunction may be the result of a failure in any one of these areas. The term "sexual dysfunction" refers to a couple's normal or typical pattern of sexual desire and/or responsiveness being persistently impaired [3].

Pain, immobility, and reduced muscular strength caused by musculoskeletal problems make it harder to perform a sexual act, reducing the amount of sexual excitement. When a person suffers from low back pain, it has an effect on their sexual activity because it causes discomfort during sexual interactions, which reduces their willingness to engage in sexual activity or changes their emotions toward their partner.

In Turkey, 55\% of men and $84 \%$ of women with lumbar disc herniation experienced sexual difficulties [5]; in Sweden, 34\% complained of additional LBP linked to sexual activity and $30 \%$ stated that their sex life was severely restricted because of LBP [6]. [5] [6] Lumbar disc herniation reduced sexual desire and frequency of sexual engagement in 50\% of the Japanese population [7]. Chronic LBP patients in Morocco (81\%) reported sexual problems as a result of their condition [2], whereas in Iran $(71.1 \%$ of females and $59.5 \%$ of men) sexual incapacity and erectile dysfunction are also common complaints [8].

In Egyptian culture, attitudes about sex and open discussion of it, particularly among women, have reached taboo levels. Previous research in our society on female sexual function and dysfunction was very rare [9].

The problem of SD in ladies with persistent low back pain has not been addressed by any researchers in our nation, as far as we know. This study aims to shed light on the problem of CLBP in Egypt's female sexual life and explain the consequences.

\section{Patients and methods}

This cross-sectional study was conducted in outpatient Clinic of Neurosurgery Department Zagazig University Hospitals. on married women complaining of chronic low back pain. The study was done throughout six months starting from March 2020 to September 2020.

\subsection{Patients' inclusion criteria:}

- Women with chronic low back pain for more than 6 months.

- Married women of all age groups with current active sexual life.

\subsection{Patients' exclusion criteria:}

- Unmarried women.

- Women who had low back pain less than 6 months. 
- Illiterate women who will not be able to fill the questionnaire by themselves.

- Women who have medical conditions preventing them from regular sexual activity e.g. mental and psychiatric diseases.

\subsection{All patients were subjected to:}

- Complete history taking and neurological examination to identify the intensity, location and duration of chronic low back pain.

- History of previous malignancies, surgical procedures, interventions or radiation.

- All patients were asked for leg pain and sciatica and subjected to leg raising test for sciatica, which was recorded either 1) not present, 2) unilateral either right or left, 3) bilateral.

- Personal history:

- The duration of complaint from low back pain by years.

- Self-report questionnaire designed by Younis et al. (2015), including 25 questions and other questions were added to suit the purpose of study. It is written in English and translated into Arabic. To ensure that all gathered information were kept confidential and the subject was anonymous, each questionnaire is handed in an open envelope and after filling it, the subject sealed the envelope and put it in a basket containing other sealed envelopes. Approval was obtained from ethical committee and the department of Dermatology, Venereology and Andrology, Benha University and from patients included in the study.

\subsection{Statistical methods}

Data management and statistical analysis were done using SPSS version 25. (IBM, Armonk, New York, United States). Numerical data were summarized as means and standard deviations. Categorical data were summarized as numbers and percentages. Quantitative scores were compared at three months and six months using paired t-test. Schatzker and Lambert score was compared at 3 and 6 months using the sign test. All statistical tests were two-sided. $\mathrm{P}$ values less than 0.05 were considered significant.

\section{Results}

Our resulted showed that $35 \%$ of the studied participants aged 30-39 years. Regarding age of marriage, $40.1 \%$ of participants married at ages less than 20 years. Regarding education, $40.1 \%$ of participants had a university degree. $54.8 \%$ had a positive history of FGC. table 1

Table (1) Distribution of the studied participants according to demographic data ( $\mathrm{n}=217)$.

\begin{tabular}{lcc}
\hline & n & \% \\
\hline Age: & 32 & 14.7 \\
$20-29$ year & 76 & 35.0 \\
$30-39$ year & 55 & 25.3 \\
$40-49$ year & 54 & 24.9 \\
250 year & & \\
Age at marriage: & 87 & 40.1 \\
<20 year & 76 & 35.0 \\
$20-29$ year & 54 & 24.9 \\
$30-39$ year & & \\
Education level: & 65 & 30.0 \\
Read and write & 65 & 30.0 \\
Secondary education & 87 & 40.1 \\
University degree & & \\
FGC: & 98 & 45.2 \\
No & 119 & 54.8 \\
Yes
\end{tabular}

Table (2) shows that among participants, 39.6\% reported that the duration of chronic low back pain was more than 10 years.

Table (2) Distribution of the studied participants according to history of back pain $(n=217)$.

\begin{tabular}{lll}
\hline & $\mathbf{n = 2 1 7}$ & \% \\
\hline Back pain: & & \\
$<1$ year & 44 & 20.3 \\
$1-5$ years & 33 & 15.2 \\
$5-10$ years & 54 & 24.9 \\
$>10$ years & 86 & 39.6 \\
\hline
\end{tabular}

Table (3) shows that there is a statistically significant association between duration of CLBP and age of patients, age at marriage and education level. 
Table (3) Relation between duration of back pain and demographic data of the studied participants.

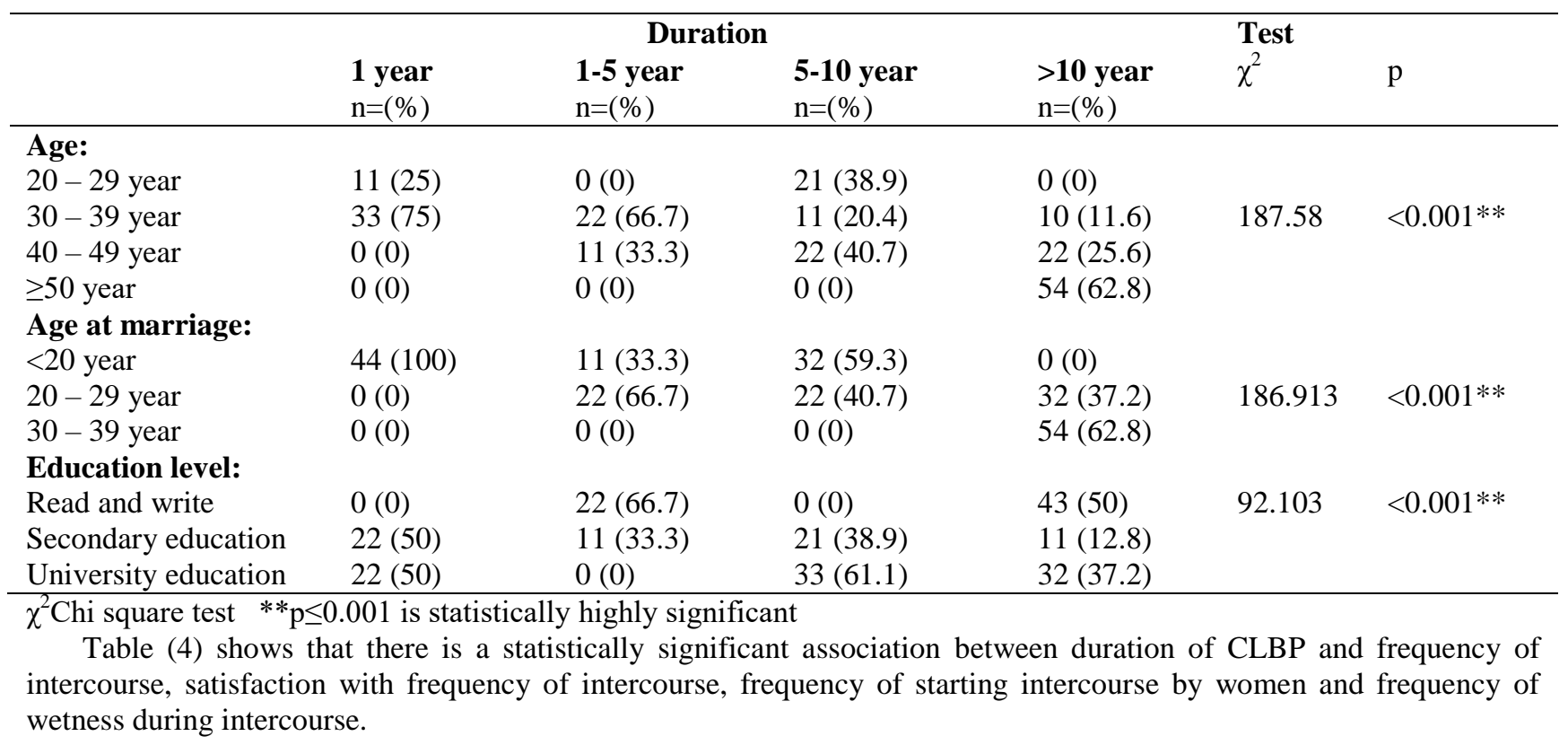

Table (4) Relation between duration of back pain and sexual history of the studied participants ( $\mathrm{n}=217$ ).

\begin{tabular}{|c|c|c|c|c|c|c|c|}
\hline & & \multicolumn{3}{|c|}{ Duration } & \multicolumn{3}{|c|}{ Test } \\
\hline & & $\begin{array}{l}1 \text { year } \\
n=44(\%)\end{array}$ & $\begin{array}{l}1-5 \text { year } \\
\mathrm{n}=33(\%)\end{array}$ & $\begin{array}{l}\text { 5-10 year } \\
\mathrm{n}=54(\%)\end{array}$ & $\begin{array}{l}>10 \text { year } \\
\mathrm{n}=86(\%)\end{array}$ & $\chi^{2}$ & $\mathrm{p}$ \\
\hline \multicolumn{8}{|c|}{$\begin{array}{ll}\text { Frequency } & \text { of } \\
\text { intercourse: } & \end{array}$} \\
\hline $2-3$ times/week & & $11(25)$ & $11(33.3)$ & $11(20.4)$ & $0(0)$ & 187.58 & $<0.001 * *$ \\
\hline Once weekly & & $33(75)$ & $11(33.3)$ & $43(79.6)$ & $22(25.6)$ & & \\
\hline Once monthly & & $0(0)$ & $11(33.3)$ & $0(0)$ & $42(48.8)$ & & \\
\hline <once monthly & & $0(0)$ & $0(0)$ & $0(0)$ & $22(25.6)$ & & \\
\hline \multicolumn{8}{|c|}{$\begin{array}{l}\text { Satisfaction with } \\
\text { frequency: }\end{array}$} \\
\hline Yes & & $33(75)$ & $22(66.7)$ & $32(59.3)$ & $32(37.2)$ & 31.565 & $<0.001 * *$ \\
\hline No, need more & & $0(0)$ & $0(0)$ & $11(20.4)$ & $22(25.6)$ & & \\
\hline No, need less & & $11(25)$ & $11(33.3)$ & $11(20.4)$ & $32(37.2)$ & & \\
\hline \multicolumn{8}{|c|}{$\begin{array}{l}\text { Frequency of starting } \\
\text { intercourse: }\end{array}$} \\
\hline Never & & $0(0)$ & $22(66.7)$ & $22(40.7)$ & $64(74.4)$ & 177.46 & $<0.001 * *$ \\
\hline $\begin{array}{l}<50 \% \text { of } \\
\text { encounters }\end{array}$ & sexual & $11(25)$ & $11(33.3)$ & $32(59.3)$ & $22(25.6)$ & & \\
\hline $\begin{array}{l}\geq 50 \% \text { of } \\
\text { encounters }\end{array}$ & sexual & $33(75)$ & $0(0)$ & $0(0)$ & $0(0)$ & & \\
\hline \multicolumn{8}{|c|}{$\begin{array}{l}\text { Frequency of wetness } \\
\text { during intercourse: }\end{array}$} \\
\hline Never & & $22(50)$ & $0(0)$ & $11(20.4)$ & $0(0)$ & & \\
\hline $\begin{array}{l}<50 \% \text { of } \\
\text { encounters }\end{array}$ & sexual & $11(25)$ & $0(0)$ & $0(0)$ & $11(12.8)$ & 92.437 & $<0.001 * *$ \\
\hline $\begin{array}{l}\geq 50 \% \text { of } \\
\text { encounters }\end{array}$ & sexual & $11(25)$ & $33(100)$ & $43(76.9)$ & $75(87.2)$ & & \\
\hline
\end{tabular}

\section{Discussion}

In Egyptian culture, attitudes about sex and open discussion of it, particularly among women, have reached taboo levels. Previous research in our society on female sexual function and dysfunction was very rare [9].

The problem of SD in ladies with persistent low back pain has not been addressed by any researchers in our nation, as far as we know. This study aims to shed 
light on the problem of CLBP in Egypt's female sexual life and explain the consequences. The Younis et al. [10] self-report questionnaire was used at the Neurosurgery Department Outpatient Clinic at Zagazig University Hospitals.

According to the results of our research, $35 \%$ of participants are between the ages of 30 and 39. In terms of marital age, $40.1 \%$ of participants were under the age of 20 when they tied the knot. In terms of education, $40.1 \%$ of participants had a bachelor's degree or above, and $54.8 \%$ had had FGC treatment in the past. Chronic low back pain was linked to decreased female erections in a study by Drummond et al. [11]. The average person was 30.31 years old, a difference of 7.10 years.

In our research, we found a statistically significant link between having had a FGC in the past and the age of the patient, the age at which the patient married, and the frequency with which the patient interacted. FGC exposure reduced frequency in $18.5 \%$ of participants, who reported satisfaction with the frequency of intercourse and frequency of wetting in the bed during intercourse ( 88 percent and 63 percent of participants unexposed and exposed respectively get wetduring intercourse in more than 50 percent of sexual encounters).

Age and health condition were shown to be substantially related with female sexual dysfunction in a study by Basok et al. [12].

Researchers Bahouq et al. [2] studied the effect of CLBP on patients' sexual lives and discovered that $81 \%$ of those with CLBP had sexual problems; no links were identified between these variables, indicating that sexuality is harmed in those with CLBP. Disturbances have an impact on both the sexual act and the quality of one's sexual life. These disruptions are linked to a slew of variables. In males and older patients, altered sexual quality of life was more frequent and was related to a reduction in the frequency of sexual intercourse rather than back pain and libido loss. Having low functional status was linked to worse sexual quality of life.

Sexual dysfunction was shown to be more common in CLBP patients than in healthy controls, according to a study by Nikoobakht et al. [8]. Patients with CLPB are more likely to have sexual dysfunction. Therefore, it has been discovered that lower female sexual function in female patients with CLBP is associated with older patients having a higher BMI, a lower educational level, a lower family income, a poor functional status, being physically inactive, being unemployed, irregular menstruation, prolonged disease and sick leave, higher levels of depression and anxiety and a poor quality of life When compared to women, male patients with advanced age, high body mass index (BMI), low education, low family income and poor functional status (such as being physically inactive or retired) are more likely to experience sexual dysfunction, as are those with chronic illnesses and long-term sick leave, as well as higher levels of depression and anxiety.

In a group of individuals with chronic pain, Gruenwald et al. [13] looked at the frequency and severity of sexual dysfunction (SD). They came to the conclusion that a large proportion of people with chronic pain had mild to moderate SD. The degree of $\mathrm{SD}$ is inversely proportional to the intensity of the accompanying pain. To find out how common and relevant sexual impairment caused by LBP is, Ferrari et al. [14] conducted research to find out how many Italian patients reported that their sexual life was affected by pain in some way, just as in other countries. When it comes to persistent low back pain and female sexual function, Drummond et al. [11] found no connection.

With so little information available, this study stands out as a valuable beginning point for future research on the issue. Women with persistent low back pain showed some impairment in sexual function, according to the results of this research. As a result, these women had low levels of sexual function despite the fact that functional activities were unaffected. By using an educational approach that involves exercising the muscles associated with physiopathology in the lower back and pelvis, such as perineal awareness and strengthening of pelvic floor musculature, the physical therapist can intervene preventatively, increasing pelvic vascularization and sensitivities while also encouraging sexual satisfaction.

Patients with persistent low back pain often have female sexual dysfunction. As a result, studies on female participants should examine sexual function to see whether it has an effect on overall well-being.

\section{Conclusion}

Female patients with persistent low back pain had a sex dysfunction prevalence of $54.8 \%$, according to the study. Chronic low back pain was linked to decreased female erections. It's obvious that doctors in this area need to be made more aware of the diagnosis and treatment of sexual issues in women with persistent low back pain.

\section{References}

[1] Y. Tang et al., "Assessment of the association between paraspinal muscle degeneration and quality of life in patients with degenerative lumbar scoliosis," Exp. Ther. Med. vol.20, pp. 505-511, 2020.

[2] H. Bahouq, A. Fadoua, R. Hanan, H. Ihsane, and H.-H. Najia, "Profile of sexuality in Moroccan chronic low back pain patients," BMC Musculoskelet. Disord.vol.14,pp. 1-6, 2013.

[3] E. B. Pasqualotto, F. F. Pasqualotto, B. P. Sobreiro, and A. M. Lucon, "Female sexual dysfunction: the important points to remember," Clinics. vol. 60, pp. 51-60, 2005.

[4] L. Brotto et al., "Psychological and interpersonal dimensions of sexual function and dysfunction," J. Sex. Med.vol. 13, pp. 538-571, 2016.

[5] N. B. Akbaş, S. Dalbayrak, D. G. Külcü, M. Yilmaz, T. Yilmaz, and S. Naderi, "Assessment of sexual dysfunction before and after surgery for 
lumbar disc herniation," J. Neurosurg. Spine.vol.13,pp.581-586, 2010.

[6] S. Berg, P. Fritzell, and H. Tropp, "Sex life and sexual function in men and women before and after total disc replacement compared with posterior lumbar fusion," spine J.vol.9, pp. 987994, 2009.

[7] M. Kanayama et al., "How does surgery affect sexual desire and activities in patients with lumbar disc herniation?," Spine (Phila. Pa. 1976).vol. 35, pp. 647-651, 2010.

[8] M. Nikoobakht, N. Fraidouni, M. Yaghoubidoust, A. Burri, and A. H. Pakpour, "Sexual function and associated factors in Iranian patients with chronic low back pain," Spinal Cord.vol. 52,pp. 307-312, 2014.

[9] I. M. A. Hassanin, Y. A. Helmy, M. M. F. Fathalla, and A. Y. Shahin, "Prevalence and characteristics of female sexual dysfunction in a sample of women from Upper Egypt," Int. J. Gynecol. Obstet..vol.108, pp. 219-223, 2010.

[10] I. Younis, F. El-Esawy, and R. Abdel-Mohsen, "Is female orgasm an earth-moving experience: an
Egyptian experience," Hum. Androl.vol.5,pp. 3744, 2015.

[11]H. F. DRUMMOND, T. S. FARCIC, N. CARVAS, C. S. BALDAN, I. F. B. MASSON, and A. F. P. MACHADO, "Correlation between chronic low back pain and female sexual function," Coluna/Columna.vol. 19, pp. 277-281, 2020.

[12]E. K. Basok, N. Atsu, M. M. Rifaioglu, G. Kantarci, A. Yildirim, and R. Tokuc, "Assessment of female sexual function and quality of life in predialysis, peritoneal dialysis, hemodialysis, and renal transplant patients," Int. Urol. Nephrol.vol. 41,pp. 473-481, 2009.

[13] I. Gruenwald, T. Adler, M. Haddad, R. Leiba, and E. Eisenberg, "Sexual Dysfunction in Patients with Chronic Pain. Urol Nephrol Open Access J 4 (6): 00147. DOI: 10.15406/unoaj. 2017.04. 00147," Sex. Dysfunct. Patients with Chronic Pain.vol.2, 2017.

[14] S. Ferrari et al., "Sexual disability in patients with chronic non-specific low back pain-a multicenter retrospective analysis," J. Phys. Ther. Sci..vol. 31 ,pp. 360-365, 2019. 